\title{
How DNA Base Pairs Escape From the Excited-State: Antiaromaticity Relief in the Picoseconds
}

\author{
Lucas José Karas, ${ }^{1}$ Chia-Hua Wu, ${ }^{1 *}$ Henrik Ottosson, ${ }^{2 *}$ Judy I-Chia Wu ${ }^{1 *}$ \\ ${ }^{1}$ Department of Chemistry, University of Houston, Houston, TX 77004, USA \\ ${ }^{2}$ Department of Chemistry, Ångström Laboratory, Uppsala University, 751 20, Uppsala, Sweden
}

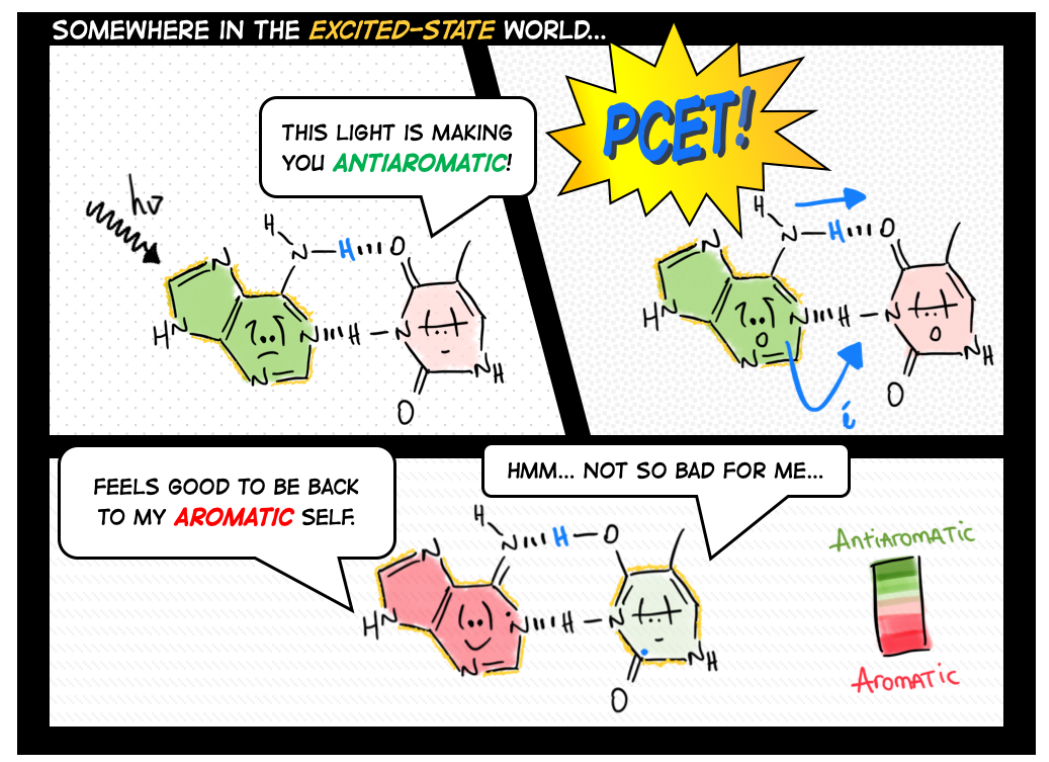

\section{Summary Paragraph}

Before the development of an ozone layer in the Archean atmosphere, the flux of UV radiation reaching Earth was suggested to be several orders of magnitude higher than it is today. For the emerging biomolecules, constant exposure to strong UV irradiation meant that useful molecules had to be resistant to UV damage and harmful photochemical reactions. From this prebiotic environment, the Watson-Crick structures of $A \cdot T$ and $G \cdot C$ base pairs survived to encode genetic information-and the photostability of these winning pairs in this specific arrangement is astonishing. ${ }^{1-3}$ Upon UV irradiation, the photoexcited canonical base pairs undergo protoncoupled electron transfer (PCET), followed by non-radiative decay, and convert internally to the electronic ground state within picoseconds. ${ }^{3-6}$ But the underlying reason why this process happens so efficiently has not been explained. Here we show that efficient photodeactivation in isolated base pairs are driven by antiaromaticity relief during PCET. According to computed nucleus independent chemical shifts, the $A \bullet T$ and $G \bullet C$ base pairs are aromatic in the electronic ground state, but the purines become highly antiaromatic in the first ${ }^{1} \pi \pi^{*}$ state, and PCET relieves this excited-state antiaromaticity. We found especially pronounced antiaromaticity relief for the major PCET pathway of isolated Watson-Crick $A \bullet T$ and $G \bullet C$ base pairs, when compared to alternative proton transfer routes or to PCET reactions in non-canonical pairs. Our findings suggest that excited-state deactivation of isolated base pairs are tied to sudden changes in aromaticity and antiaromaticity within the picoseconds that follow a strike of UV-light. 


\section{Main Text}

The Watson-Crick structures of $A \cdot T$ and $G \cdot C$ base pairs appear to be evolutionarily privileged because of their astonishing photostability under conditions at the time of the origin of life. When irradiated by UV-light, DNA base pairs undergo efficient excited-state deactivation at a rate too fast for other reactions to take place, and this mechanism protects the integrity of DNA. ${ }^{3-7}$ Noncanonical conformers of $A \bullet T$ and $G \cdot C$ have been shown to display much longer excited-state lifetimes. ${ }^{8}$ We now wish to suggest an explanation for the special photostability of Watson-Crick $A \cdot T$ and $G \cdot C$ base pairs.

It is understood that when isolated Watson-Crick structures of $A \cdot T$ and $G \cdot C$ are irradiated by short-wavelength light, they do not cross to a reactive triplet state, but convert internally to the electronic ground state through non-radiative decay-i.e., via a "Domcke channel". ${ }^{4-6,9,10}$ Within picoseconds, the locally excited (LE) ${ }^{1} \pi \pi^{*}$ state connects to a charge-transfer (CT) state via proton-coupled electron transfer (PCET), i.e., an electron transfers from the purine (A or $\mathrm{G}$ ) to the pyrimidine ( $\mathrm{T}$ or $\mathrm{C}$ ) and a proton follows. From there, the charge-transferred structure passes through a conical intersection $(\mathrm{Cl})$, and returns to the electronic ground state (GS) (Figure 1). Gas-phase experiments recorded short excited-state lifetimes for the isolated Watson-Crick structures of $A \cdot T$ (190 ps) and $G \cdot C$ (40 ps) base pairs. ${ }^{11}$ We propose that the reason for this efficient excited-state deactivation is a sudden change in the aromatic and antiaromatic character of isolated base pairs. In the ground state, the $A \cdot T$ and $G \bullet C$ base pairs are aromatic, but when struck by UV-light the purines become antiaromatic, and PCET is the escape route to relieve excited-state antiaromaticity.

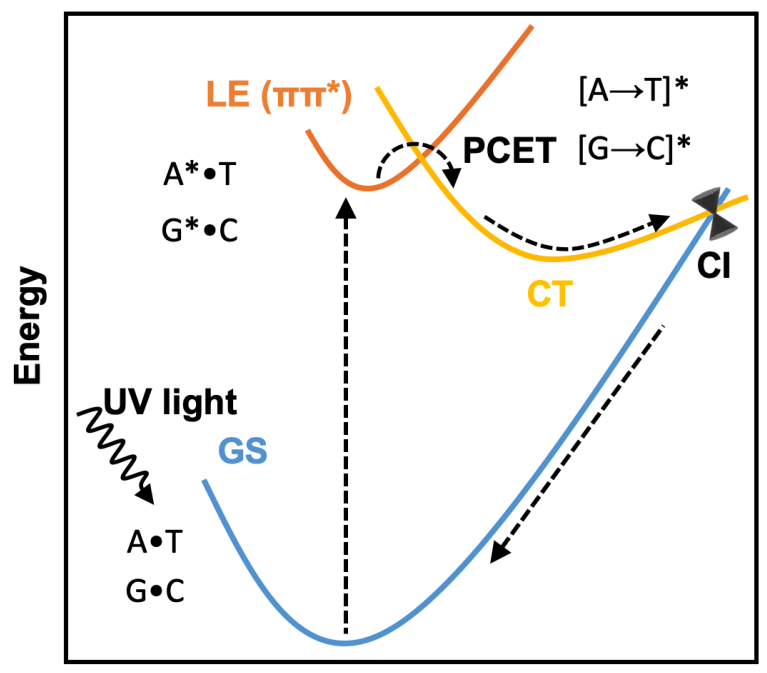

Reaction Coordinate

Figure 1. Schematic illustration of excited-state deactivation in DNA base pairs. 
Hückel's $1931^{12}$ paper first proposed a theory to determine the aromatic and antiaromatic characters of compounds using an electron-counting method. He suggested that closed-shell, cyclic, m-conjugated, organic compounds with [4n+2] ring m-electrons exhibit aromatic character, and that those with [4n] ring $\pi$-electrons display antiaromatic character. On this basis, the purines ( $A$ and $G$, ten ring $\pi$-electrons) and pyrimidines ( $T$ and $C$, six ring $\pi$-electrons) in DNA are formally aromatic; the $\mathrm{C}=\mathrm{O} \pi$-bonds can be considered in their charge separated resonance forms $\mathrm{C}\left(\delta^{+}\right)-\mathrm{O}\left(\delta^{-}\right)$since the $\pi$-electrons are polarized towards the $\mathrm{O}$ atom. Baird suggested that these electron-counting rules reverse at the lowest excited ${ }^{3} \pi \pi^{*}$ states; ${ }^{13}$ compounds with [4n] ring $\pi$-electrons are aromatic and those with [4n+2] ring m-electrons are antiaromatic. Later works found Baird's rule to extend also to the first ${ }^{1} \pi \pi^{*}$ states of organic compounds ${ }^{14-17}$ and has significant interpretive merit for the photochemistry of organic compounds. ${ }^{18}$ In the ${ }^{1} \pi \pi^{*}$ state, the A, T, G, C bases are [4n+2] m-antiaromatic. We now show that these features can explain important experimental observations of excited-state deactivation in isolated canonical and noncanonical $A \cdot T$ and $G \cdot C$ base pairs.

We computed nucleus independent chemical shifts, NICS(1)zz, to quantify the aromatic and antiaromatic characters of bases pair structures at relevant planar $\left(C_{s}\right)$ equilibrium geometries in the ground state (GS), ${ }^{1} \pi{ }^{*}$ locally excited state (LE), and charge-transfer state (CT). NICS $(1)_{z z}$ values are magnetic shielding tensors computed in the form of "ghost atoms" at $1 \AA$ above ring centers, and reversed in sign to match experimental conventions for chemical shifts. ${ }^{19-21}$ Computed NICS at the ring centers of individual bases in base pairs were performed at the $\operatorname{CASSCF}(10,10) / 6-311+G(d, p)$ level, based on base pair geometries optimized at (TD) $\omega B 97 X-D / 6-311+G(d, p)$ (see full methods in the Supplementary Information, SI). Negative NICS(1) zz values indicate aromaticity (diatropicity), positive NICS(1)zz values indicate antiaromaticity (paratropicity). Especially large positive NICS values are an artifact of the NICS method, and can occur for antiaromatic molecules with significant multiconfigurational character. Evaluations of aromaticity and antiaromaticity based on the multicenter index $(\mathrm{MCl})$ method, ${ }^{22}$ an electronic index for aromaticity, are included in the SI for comparison.

Computed NICS $(1)_{z z}$ for the Watson-Crick $A \cdot T$ and $G \cdot C$ base pairs show that the purines ( $A$ and $G$ ) are aromatic and the pyrimidines ( $T$ and $C$ ) are weakly aromatic in the electronic ground state (see Figure 2). But upon photoexcitation, the purines become highly antiaromatic in the ${ }^{1} \pi \pi^{*}$ LE state; note large positive $\operatorname{NICS}(1)_{z z}$ for the $A^{*}$ (see $A^{*} \cdot T$ in Figure $2 A$ ) and $G^{*}$ (see $G^{*} \cdot C$ in Figure $2 B$ ) fragments. Crossing to the $C T$ state relieves antiaromaticity. Following a barrierless PCET reaction, the photoexcited purines lose an electron and a proton to the pyrimidines and regain aromatic character in the CT state. This re-aromatization stabilizes the CT state structures, $[A \rightarrow T]^{*}(3.2 \mathrm{eV}$, relative to ground state $A \bullet T)$ and $[G \rightarrow C]^{*}(2.6 \mathrm{eV}$, relative to ground state $G \cdot C$ ). As a result, the $S_{1}$ and $S_{0}$ surfaces come close and a canonical intersection can happen. 
Potential energy curves for the LE and CT states were computed based on constrained geometry optimizations, in which the proton transferring $\mathrm{N}-\mathrm{H}$ bonds were set to distances between $1 \AA$ and $2.5 \AA$, and varied at increments of $0.1 \AA$. Ground state potential energy curves were computed based on single point energies of the corresponding optimized CT state geometries. All energy points on the GS, LE, and CT curves were computed at CASPT2 $(8,8) / 6$ $311+G(d, p)$ (see full methods in the $S I)$.
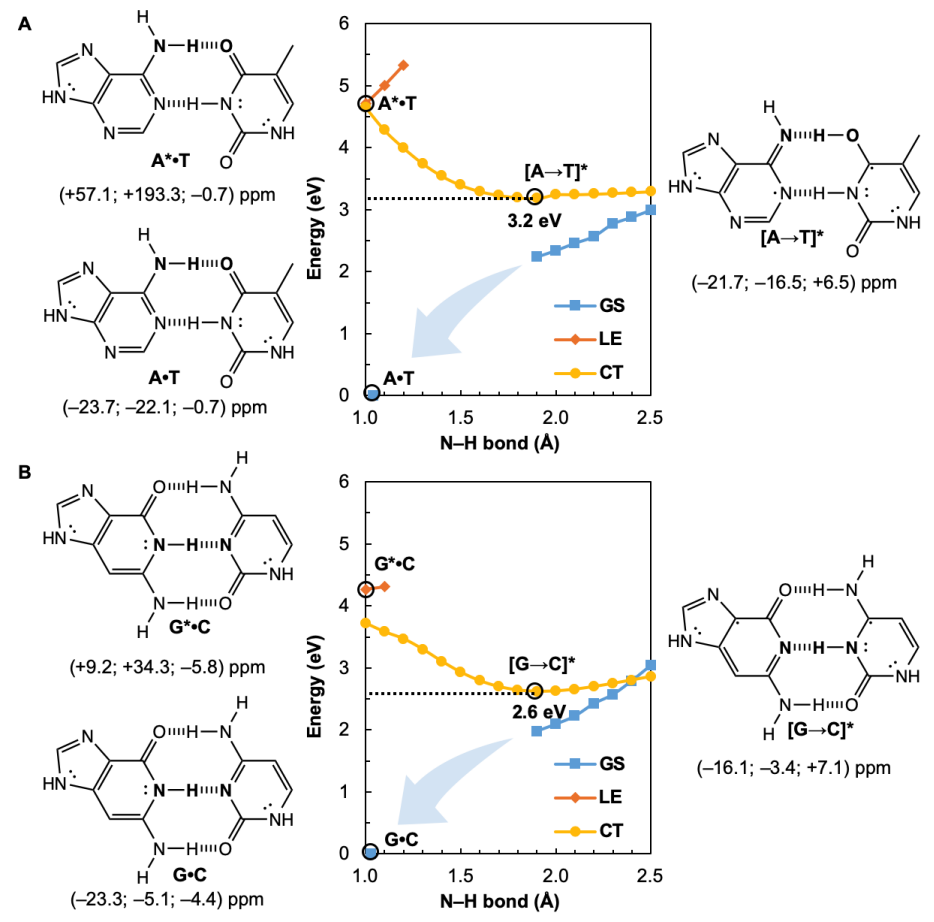

Figure 2. Potential energy functions of the electronic ground state (GS), ${ }^{1} \pi \pi^{*}$ locally-excited state (LE), and charge-transfer state (CT), with constrained $\mathrm{N}-\mathrm{H}$ bond distance (see $\mathrm{N}-\mathrm{H}$ in bold), for the Watson-Crick A) $A \cdot T$ and B) $G \cdot C$ structures, at CASPT2 $(8,8) / 6-311+G(d, p)$. $\operatorname{NICS}(1)_{z z}$ values were computed at $\operatorname{CASSCF}(10,10) / 6-311+G(d, p)$ for equilibrium structures.

We wondered whether a similar mechanism could explain why PCET in the reversed direction (i.e., electron transfer and proton transfer from a photoexcited pyrimidine to the paired purine) is absent in Watson-Crick base pairs. Theoretical evidence have shown that PCET cannot happen when excitation takes place on the pyrimidines of isolated canonical base pairs; ${ }^{23,24}$ crossing between the first ${ }^{1} \mathrm{Tm}^{*}$ state and the CT state involves a high barrier to PCET. We found that when excitation happens on the pyrimidines ( $T$ and $C$ ), they only become modestly antiaromatic (since these rings have breached ring m-electron delocalizations and are near-nonaromatic in the ground state), while the purines remain largely aromatic. Note the small positive 
NICS(1) zz values for $T^{*}$ and $C^{*}$, but negative NICS(1)zz values for $A$ and $G$, in the LE state structures of $A \cdot T^{*}$ (Figure $3 A$ ) and $G \cdot C^{*}$ (Figure $3 B$ ). When and if PCET happens, the purported equilibrium CT state structures, $[A \leftarrow T]^{*}(5.2 \mathrm{eV}$ relative to ground state $A \bullet T)$ and $[G \leftarrow C]^{*}(4.9 \mathrm{eV}$ relative to ground state $G \cdot C$ ), are relatively high in energy since the purines gain an electron (11 ring m-electrons) and lose aromatic character (Figure 3). PCET is disfavored, since there is less drive to relieve antiaromaticity in the LE state, and the CT state structure is not especially stabilized by aromatic character in the purines or pyrimidines. This may explain why a PCET deactivation route is not viable for the locally excited Watson-Crick structures of $A \cdot T^{*}$ and $G \cdot C^{*}$, why these reactive states must deactivate through other pathways, ${ }^{11}$ and possibly why DNA base pair damage often happens on the pyrimidines, like thymine. ${ }^{7}$
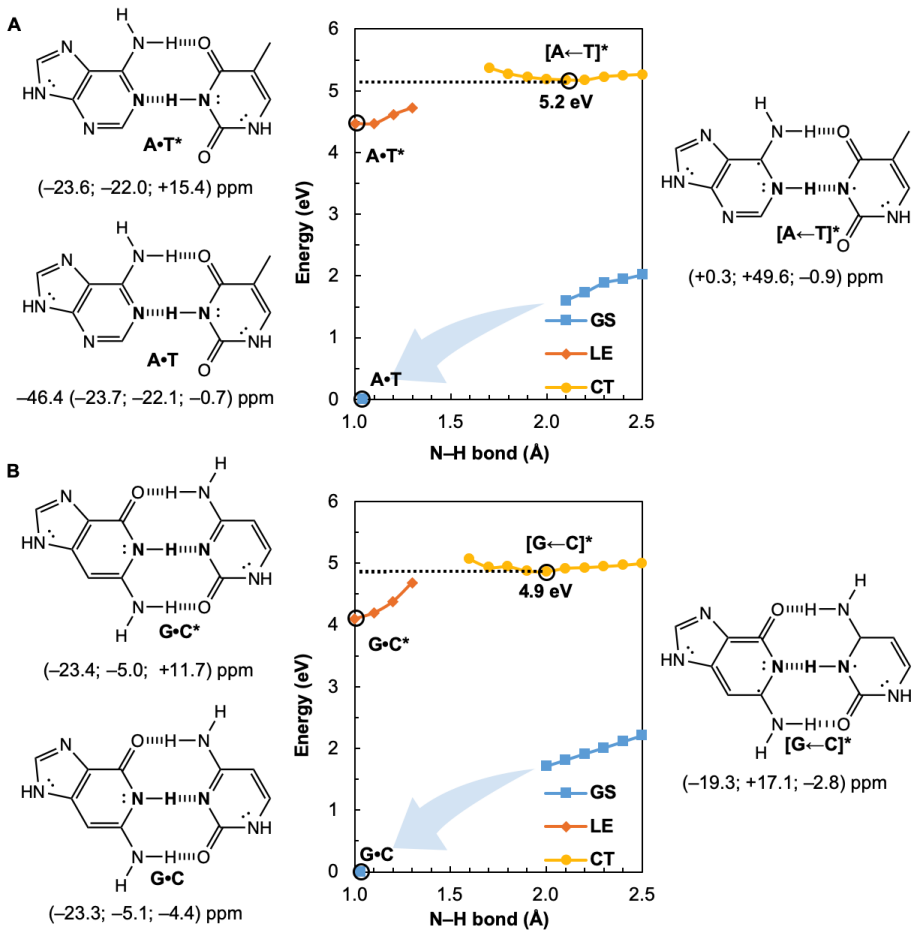

Figure 3. Potential energy functions of the electronic ground state (GS), locally-excited ${ }^{1} \pi \pi^{*}$ state (LE), and charge-transfer state (CT), with constrained $\mathrm{N}-\mathrm{H}$ bond distance (see $\mathrm{N}-\mathrm{H}$ in bold), for the Watson-Crick structures of $A$ ) $A \bullet T$ and $B$ ) $G \cdot C$, with local excitations on pyrimidines, and computed NICS(1)zz values for the equilibrium structures.

Are the Watson-Crick arrangements special? Experiments have found the isolated Watson-Crick G•C pair to exhibit a broad UV peak, compared to sharp peaks for alternative arrangements, and suggesting a short excited-state lifetime. ${ }^{8}$ IR-UV spectra for several isomers 
of isolated $A \cdot T$ pairs were found to match well with computed IR spectra of the supposed structures. ${ }^{25}$ But that of the Watson-Crick pair matched poorly (resembling the spectra for a Hoogsteen pair instead), possibly due to a short excited-state lifetime. Computational evidence showed that many isolated non-canonical $A \bullet T$ and $G \bullet C$ base pair arrangements lacked the required conical intersection for rapid excited-state deactivation. ${ }^{5,6}$ We considered the most stable non-Watson-Crick (non-WC) conformers of $A \cdot T^{25}$ and $G \cdot C^{5}$ and found the purines to be antiaromatic in the ${ }^{1} \pi \pi^{*}$ LE states-just like the Watson-Crick forms (Figure 4). But when PCET happens, the equilibrium CT state structures, non-WC-[A $\rightarrow T]^{*}$ and non-WC- $[\mathrm{G} \rightarrow \mathrm{C}]^{*}$, are stabilized by aromaticity to a lesser extent; the purine rings show only weak to modest aromatic character, but the pyrimidines are antiaromatic. As a result, these non-canonical CT state structures are higher in energy: non-WC- $[\mathrm{A} \rightarrow \mathrm{T}]^{*}(4.6 \mathrm{eV}$ relative to ground state non-WC-A•T$)$ and non-WC-[G $\rightarrow C]^{*}(3.5 \mathrm{eV}$ relative to ground state non-WC-G•C) (cf. relative energy of $C T$ structure for the Watson-Crick conformers in Figure 2), and a conical intersection is less likely to happen.
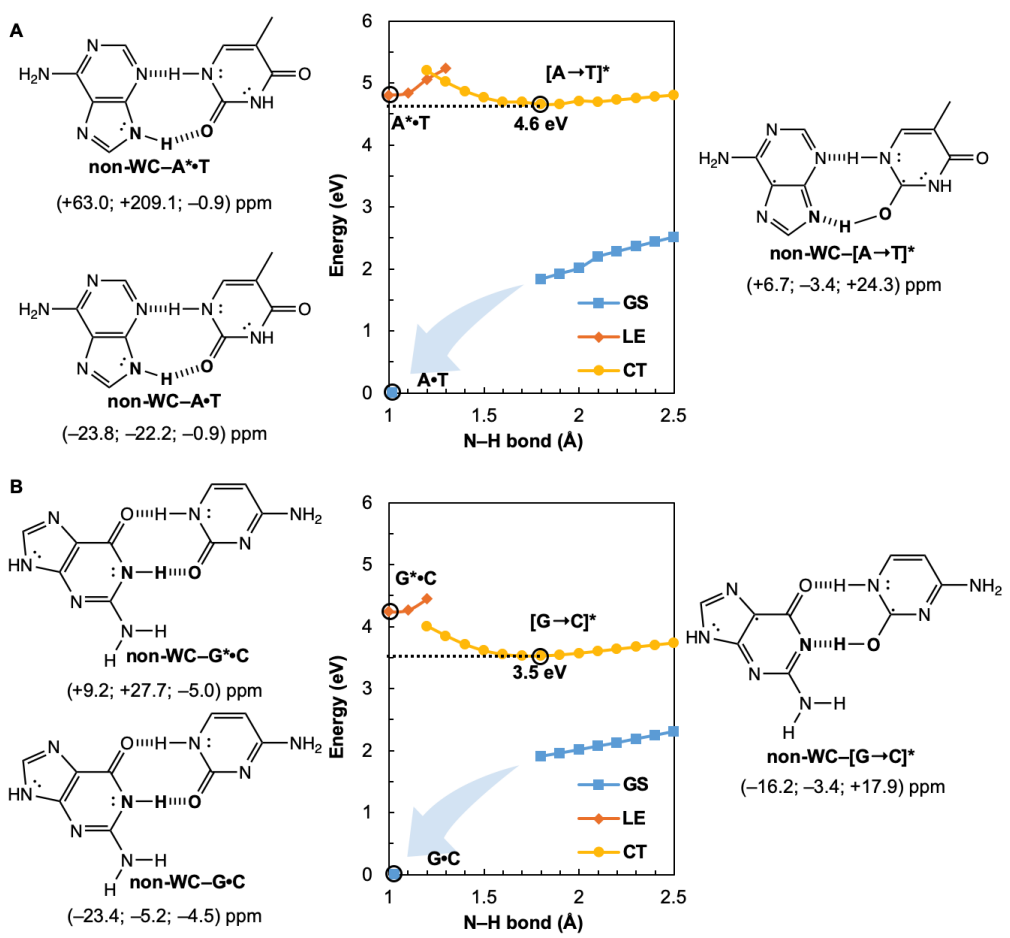

Figure 4. Potential energy functions of the electronic ground state (GS), locally-excited ${ }^{1} \pi \pi^{*}$ state (LE), and charge-transfer state (CT), with constrained $\mathrm{N}-\mathrm{H}$ bond distance, for the most stable non-Watson-Crick forms of $\mathbf{A}) \mathrm{A} \bullet T$ and $\mathbf{B}) \mathrm{G} \cdot \mathrm{C}$, and computed NICS(1)zz values for the equilibrium structures. 
It is tempting to imagine that textbook concepts like aromaticity and antiaromaticity may have played a decisive role in the molecular evolution of bases and base pairs towards encoding genetic information. The best-documented PCET pathway of double-stranded DNA involves intrastrand electron transfer between stacked nucleobases, followed by inter-strand proton transfer in the resulting radical anion base pair. ${ }^{26}$ But the role of excited-state antiaromaticity we put forth for explaining photodeactivation in isolated base pairs may very well apply. When light strikes a DNA base pair, the purines become excited-state antiaromatic, and to escape from being "excitedstate antiaromatic" an electron must go away-either by departing to an immediate hydrogen bonded pair or to a neighboring stacked base. We notice that the role of excited-state antiaromaticity also may provide mechanistic insights to other light-driven proton or electron transfer reactions.

\section{References}

1. Boldissar, S. \& de Vries, M. S. How nature covers its bases. Phys. Chem. Chem. Phys. 20, 9701-9716 (2018).

2. Beckstead, A. A.; Zhang, Y.; de Vries, M. S.; Kohler, B. Life in the light: nucleic acid photoproperties as a legacy of chemical evolution. Phys. Chem. Chem. Phys. 18, 24228-24238 (2016).

3. Middleton, C. T., de La Harpe, K., Su, C., Law, Y. K., Crespo-Hernández, C. E. \& Kohler, B. DNA excited-state dynamics: from single bases to the double helix. Annu. Rev. Phys. Chem. 60, 217-239 (2009).

4. Schultz, T., Samoylova, E., Radloff, W., Hertel, I. V., Sobolewski, A. L. \& Domcke, W. Efficient deactivation of a model base pair via excited-state hydrogen transfer. Science 306, 1765-1768 (2004).

5. Sobolewski, A. L., Domcke, W. \& Hättig, C. Tautomeric selectivity of the excited-state lifetime of guanine/cytosine base pairs: the role of electron-driven proton-transfer processes. Proc. Natl. Acad. Sci. U. S. A. 102, 17903-17906 (2005).

6. Perun, S., Sobolewski, A. L. \& Domcke, W. Role of electron-driven proton-transfer processes in the excited-state deactivation of the adenine-thymine base pair. J. Phys. Chem. A 110, 90319038 (2006).

7. Schreier, W. J., Gilch, P. \& Zinth, W. Early events of DNA photodamage. Annu. Rev. Phys. Chem. 66, 497-519 (2015).

8. Abo-Riziq, A., Grace, L., Nir, E., Kabelac, M., Hobza, P. \& de Vries, M. S. Photochemical selectivity in guanine-cytosine base-pair structures. Proc. Natl. Acad. Sci. U.S.A. 102, 20-23 (2005).

9. Sobolewski, A. L. \& Domcke, W. Ab initio studies on the photophysics of the guanine-cytosine base pair. Phys. Chem. Chem. Phys. 6, 2763-2771 (2004).

10. Groenhof, G., Schäfer, L. V., Boggio-Pasqua, M., Goette, M., Grubmüller, H. \& Robb, M. A. Ultrafast deactivation of an excited-state cytosine-guanine base pair in DNA. J. Am. Chem. Soc. 
129, 6812-6819 (2007).

11. Bucher, D. B., Schlueter, A., Carell, T. \& Zinth, W. Watson-Crick base pairing controls excited-state decay in natural DNA. Angew. Chem. Int. Ed. 53, 11366-11369 (2014).

12. Hückel, E. Quantentheoretische Beiträge zum Benzolproblem I. Die Elektronenkonfiguration des Benzols und verwandter Verbindungen, Z. Phys. 70, 204-286 (1931).

13. Baird, N. C. Quantum organic photochemistry. II. Resonance and aromaticity in the lowest ${ }^{3} \pi \pi^{*}$ state of cyclic hydrocarbons. J. Am. Chem. Soc. 94 4941-4948 (1972).

14. Aihara, J.-I. Aromaticity-based theory of pericyclic reactions. Bull. Chem. Soc. Jpn. 51, 17881792 (1978).

15. Karadakov, P. B. Ground- and excited-state aromaticity and antiaromaticity in benzene and cyclobutadiene. J. Phys. Chem. A 112, 7303-7309 (2008).

16. Karadakov, P. B. Aromaticity and Antiaromaticity in the Low-Lying Electronic Ground States of Cyclooctatetraene. J. Phys. Chem. A 112, 12707-12713 (2008).

17. Feixas, F., Vandenbussche, J., Bultinck, P., Matito, E. \& Solà M. Electron delocalization and aromaticity in low-lying excited states of archetypal organic compounds. Phys. Chem. Chem. Phys. 13, 20690-20703 (2011).

18. Rosenberg, M., Dahlstrand, C., Kilså K. and Ottosson H. Excited state aromaticity and antiaromaticity: opportunities for photophysical and photochemical rationalizations. Chem. Rev. 114, 5379-5425 (2014).

19. Chen, Z., Wannere, C. S., Corminboeuf, C., Puchta, R. \& Schleyer, P. v. R. NucleusIndependent Chemical Shifts (NICS) as an aromaticity criterion. Chem. Rev. 105, 3842-3888 (2005).

20. Corminboeuf, C., Heine, T., Seifert, G., Schleyer, P. v. R. \& Weber, J. Induced magnetic fields in aromatic [n]-annulenes-interpretation of NICS tensor components. Phys. Chem. Chem. Phys. 6, 273-276 (2004).

21. Gogonea, V., Schleyer, P. v. R. \& Schreiner, P. R. Consequence of triplet aromaticity in $4 n \pi-$ electron annulenes: calculation of magnetic shieldings for open-shell species. Angew. Chem. Int. Ed. 37, 1945-1948 (1998).

22. Bultinck, P., Ponec, R. \& van Damme, S. Multicenter bond indicies as a new measure of aromaticity in polycyclic aromatic hydrocarbons. J. Phys. Org. Chem. 18, 706-718 (2005).

23. Gobbo, J. P., Saurí, V., Roca-Sanjuán, D., Serrano-Andrés, L., Merchán, M. \& Borin, A. C. On the deactivation mechanisms of adenine-thymine base pair. J. Phys. Chem. B 116, 4089-4097 (2012).

24. Sauri, V., Gobbo, J. P., Serrano-Pérez, J. J., Lundberg, M., Coto, P. B., Serrano-Andrés, L., Borin, A. C., Lindh, R., Merchán M. \& Roca-Sanjuán, D. Proton/hydrogen transfer mechanisms in the guanine-cytosine base pair: photostability and tautomerism. J. Chem. Theory Comput. 9, 481-496 (2013).

25. Plutzer, C.; Hunig, I.; Kleinermanns, K.; Nir, Eyal; de Vries, M. S. Pairing of Isolated Nucleobases: Double Resonance Laser Spectroscopy of Adenine-Thymine. ChemPhysChem, 8, 838-842 (2003). 
26. Zhang, Y.; de la Harpe, K.; Beckstead, A. A.; Improta, R. \& Kohler, B. UV-Induced proton transfer between DNA strands. J. Am. Chem. Soc. 137, 7059-7062 (2015).

\section{Acknowledgements}

J. I. W. thanks the National Science Foundation (CHE-1751370) and the National Institute of General Medical Sciences (NIGMS) of the National Institute of Health (R35GM133548) for grant support, as well as computational resources provided by the uHPC cluster, managed by the University of Houston and acquired through support from the NSF (MRI-1531814). H. O. thanks the Swedish Research Council for grant support (2015-04538). 